\title{
Association between neuroendocrine tumors biomarkers and primary tumor site and disease type based on total ${ }^{68}$ Ga-DOTATATE-Avid tumor volume measurements
}

\author{
Amit Tirosh ${ }^{1,2}$, Georgios Z Papadakis ${ }^{3,4}$, Corina Millo3, Samira M Sadowski, \\ Peter Herscovitch ${ }^{3}$, Karel Pacak', Stephen J Marx', Lily Yang ${ }^{6}$, Pavel Nockel ${ }^{6}$, \\ Jasmine Shell ${ }^{6}$, Patience Green ${ }^{6}$, Xavier M Keutgen ${ }^{6,7}$, Dhaval Patel ${ }^{6}$, Naris Nilubol ${ }^{6}$ \\ and Electron Kebebew ${ }^{6,8}$ \\ 'Section on Medical Neuroendocrinology, Eunice Kennedy Shriver National Institute of Child Health and \\ Human Development, National Institutes of Health, Bethesda, Maryland, USA, ${ }^{2}$ Sackler Faculty of \\ Medicine, Tel Aviv University, Tel Aviv, Israel, ${ }^{3}$ PET-Department, National Institutes of Health Clinical \\ Center, Bethesda, Maryland, USA, ${ }^{4}$ Institute of Computer Science (ICS), Foundation for Research and \\ Technology Hellas (FORTH), Crete, Greece, ${ }^{5}$ Endocrine and Thoracic Surgery, University Hospitals of \\ Geneva, Geneva, Switzerland, ${ }^{6}$ Endocrine Oncology Branch, National Cancer Institute, National \\ Institutes of Health, Bethesda, Maryland, USA, ${ }^{7}$ Department of Surgery, Rush University Medical Center, \\ Chicago, Illinois, USA, and ${ }^{8}$ Department of Surgery, The George Washington University, School of \\ Medicine and Health Sciences, Washington, District of Columbia, USA
}

Correspondence should be addressed to E Kebebew Email electron.kebebew@nih.gov

\begin{abstract}
Objective: To determine the association between neuroendocrine tumor (NET) biomarker levels and the extent of disease as assessed by ${ }^{68} \mathrm{Ga}$ DOTATATE PET/CT imaging.

Design: A retrospective analysis of a prospective database of patients with NETs.

Methods: Fasting plasma chromogranin A (CgA), neuron-specific enolase (NSE), gastrin, glucagon, vasoactive intestinal peptide (VIP) and pancreatic polypeptide (PP), and 24-h urinary 5-hydroxyindoleacetic acid (5-HIAA) levels were measured. Correlation between biomarkers and total ${ }^{68} \mathrm{Ga}$-DOTATATE-avid tumor volume (TV) was analyzed. Results: The analysis included 232 patients. In patients with pancreatic NETs $(n=112),{ }^{68}$ Ga-DOTATATE TV correlated with CgA $\left(r=0.6, P=0.001\right.$, Spearman). In patients with multiple endocrine neoplasia type $1(n=39)$, ${ }^{68} \mathrm{Ga}$-DOTATATE TV correlated with glucagon $(r=0.5, P=0.01)$ and PP levels $(r=0.5, P=0.049)$. In patients with von Hippel-Lindau $(n=24)$, plasma VIP $(r=0.5, P=0.02)$ and PP levels $(r=0.7, P<0.001)$ correlated with ${ }^{68} \mathrm{Ga}$-DOTATATE TV. In patients with small intestine NET (SINET, $n=74),{ }^{68} \mathrm{Ga}$-DOTATATE TV correlated with CgA $(r=0.5, P=0.02)$ and 5 -HIAA levels $(r=0.7, P<0.001)$, with 5 -HIAA $\geq 8.1 \mathrm{mg} / 24 \mathrm{~h}$ associated with metastatic disease with high positive $(81.8 \%)$ and negative $(85.7 \%)$ predictive values $(P=0.001) .{ }^{68} \mathrm{Ga}$-DOTATATE TV in patients with NET of unknown primary $(n=16)$ and those with NET of other primary location $(n=30)$ correlated with 5-HIAA levels $(r=0.8, P=0.002$ and $r=0.7$, $P=0.02$ respectively).

Conclusions: Our data supports the use of specific NET biomarkers based on the site of the primary NET and the presence of hereditary syndrome-associated NET. High urinary 5-HIAA levels indicate the presence of metastatic disease in patients with SINET.

European Journal of

Endocrinology

(2017) 176, 575-582
\end{abstract}

() 2017 European Society of Endocrinology Printed in Great Britain
Published by Bioscientifica Ltd. 


\section{Introduction}

Most neuroendocrine tumors (NETs) originate from endocrine cells spread throughout the respiratory and gastrointestinal tracts. Although these tumors tend to have an indolent natural course, NETs present with distant metastases in $40-50 \%$ of patients $(1,2,3)$, usually to the liver and regional lymph nodes (LN), and less often to the lungs, brain, bones and peritoneal cavity (1).

Tumor biomarkers are important for diagnosis and follow-up of patients with NETs, especially after treatment of locally advanced and/or metastatic disease. Several biomarkers are used for NET surveillance, including plasma levels of chromogranin A (CgA), pancreatic polypeptide (PP), neuron-specific enolase (NSE) and urinary 24-h 5-hydroxyindoleacetic acid (5-HIAA) levels depending on the primary tumor location $(4,5)$. The sensitivity and specificity of different biomarkers depend on the site of the primary tumor, with CgA mainly used for patients with pancreatic and midgut NET, NSE being most useful in patients with poorly differentiated NET and 5-HIAA in patients with carcinoid syndrome. The accuracy of biomarker measurement depends on other factors unrelated to the tumor, such as kidney function and presence of gastric atrophy for $\mathrm{CgA}$ and dietary factors and medical treatment for 5-HIAA (6). Furthermore, the determination of whether elevated NET biomarkers accurately reflect the presence of disease has been limited by the 'gold standard test' used for assessing the presence of disease; pathologic analysis and/or the type of imaging modality. As a result of these limitations, there is a lack of consensus on the utility of these biomarkers for estimating the total tumor burden in patients with NETs (7).

Well-differentiated NETs typically express somatostatin receptors (SSTRs); thus, radiolabeled somatostatin analogues have been used for diagnosis and follow-up of patients with NET (5). ${ }^{111}$ In-pentetreotide (Octreoscan) has been widely used for NET imaging, but is limited by its low image resolution and long scan duration and relatively lower SSTRs affinity (8). In contrast, the ${ }^{68} \mathrm{Gallium}\left({ }^{68} \mathrm{Ga}\right)$-DOTA-compounds recently introduced into clinical practice $\left({ }^{68} \mathrm{Ga}\right.$-DOTATATE, ${ }^{68} \mathrm{Ga}$-DOTANOC and ${ }^{68} \mathrm{Ga}$-DOTATOC) have been shown to have higher sensitivity for detecting NET than Octreoscan (9) and significantly influence the management of patients with $\operatorname{NET}(10,11)$.

To our knowledge, no study has yet assessed the utility of biochemical biomarkers for evaluating NET burden using the new-generation DOTA-peptide-based PET/CT imaging $(12,13)$. Thus, in this study, we analyzed the correlation between biochemical biomarkers of NETs and total ${ }^{68} \mathrm{Ga}$-DOTATATE-avid tumor volume (TV), as a measure of total tumor burden in patients with NET.

\section{Subjects and methods}

\section{Study population}

Patients known to have NETs based on imaging (CT, MRI and ${ }^{18} \mathrm{~F}$-FDG PET), biochemical evidence of NETs and/ or pathologically confirmed NET were included in this study. The study was performed under an investigational new drug approval from the United States Food and Drug Administration. The full study eligibility criteria were as previously reported (10). The study was approved by the National Cancer Institute Institutional Review Board and the National Institutes of Health Radiation Safety Committee (NCT01967537). Written informed consent was obtained from all study participants.

NETs were sub-grouped according to the primary tumor location based on conventional anatomic imaging, ${ }^{68} \mathrm{Ga}$-DOTATATE PET/CT and/or pathological evaluation. Patients were subdivided into those with pancreatic NETs (PNETs) or small intestine NETs (SINETs). Subjects with metastatic NET and with pathologic ${ }^{68} \mathrm{Ga}$-DOTATATE uptake but no clear primary lesion were defined as NET of unknown primary (NEToUP, $n=16$ ). Thirty subjects with NET of other origins were grouped as NET of other primary location (NEToOL) due to their small number. Duodenal NETs were included in NEToOL due to their distinct characteristics compared with other SINET (14). Tumor grade was determined according to the 2010 World Health Organization (WHO) definitions as G1, G2 or G3 according to Ki-67 index $(<3,3-20$ and $>20 \%)$ and mitotic rate $(<2,2-20$ and $>20$ per 10 high-power microscopic fields) in patients who had their tumor resected or biopsied respectively (15).

\section{Biochemical and imaging evaluation}

All patients underwent testing for fasting plasma CgA, PP, NSE, vasoactive intestinal peptide (VIP), gastrin and glucagon and 24-h 5-HIAA urinary collections. Thirtyone patients receiving chronic treatment with protonpump inhibitors and one patient with a plasma creatinine $>2 \mathrm{mg} / \mathrm{dL}$ were excluded from the analysis of plasma $\mathrm{CgA}$ levels, as they may have an increase in plasma CgA levels that does not reflect disease burden. Plasma glucagon 
levels (LINCOplex Kit, Luminex 200, MO, USA), plasma VIP levels (Peninsula Laboratories, CA, USA) and plasma PP levels (Mayo Medical laboratories, Rochester, MN, USA) (16) were measured using an immunoradiometric assay, whereas immunochemiluminometric assays were used for measuring plasma chromogranin A (Mayo Medical Laboratories), gastrin (Immulite 2000, Siemens) and NSE levels (NSE Kryptor, BRAHMS, Germany). Urinary 5-HIAA levels were measured using liquid chromatographytandem mass spectrometry (Mayo Medical Laboratories) (17). The cut-offs for positivity were determined by the kit manufacturer or by the laboratory performing the tests.

Both ${ }^{68} \mathrm{Ga}$-DOTATATE PET/CT scans and biomarkers level measurements were performed concurrently at study inclusion. The biochemical evaluation for patients with a clinical suspicion of insulinoma included measurements of fasting glucose, insulin, proinsulin and C-peptide during hypoglycemia. Supervised fasting test was conducted if necessary.

For ${ }^{68} \mathrm{Ga}$-DOTATATE PET/CT imaging, $185 \mathrm{MBq}$ $(5 \mathrm{mCi}){ }^{68} \mathrm{Ga}$-DOTATATE was administered through a peripheral intravenous line. After approximately $60 \mathrm{~min}$, the patient was positioned supine in a PET/CT scanner, and images were obtained from mid-thighs to the top of the skull. A low-dose, non-contrast-enhanced CT was used for attenuation correction and anatomic localization. Maximum standardized uptake values (SUVmax) of visualized lesions were calculated based on patient total body weight. Patients treated with longacting somatostatin analogues were scanned before the next scheduled monthly dose, whereas those on shortacting somatostatin analogues discontinued the drug $24 \mathrm{~h}$ before imaging.

\section{Quantification of tumor volume by ${ }^{68} \mathrm{Ga}$-DOTATATE $\mathrm{PET} / \mathrm{CT}$ imaging}

Disease burden was assessed by quantifying ${ }^{68} \mathrm{Ga}$-DOTATATE uptake using the MIM Vista workstation (version 6.5.9). A VOI (volume of interest) encompassing the entire scan was drawn, and subsequently, a SUVmax threshold-based approach was applied to include all sites of non-physiologic uptake. The SUV threshold-based technique for segmentation is commonly used and was tested in many clinical studies (18). The software used in the current analysis enables automatic generation of separate VOIs encircling all areas above the SUVmax threshold set by the user. This software has been tested and reported before (19). Afterward, an experienced nuclear medicine physician who was blinded to the clinical and laboratory data manually excluded areas with physiologic ${ }^{68} \mathrm{Ga}$-DOTATATE uptake. The volumes of all lesions with a pathological ${ }^{68} \mathrm{Ga}$-DOTATATE uptake were obtained automatically. The sum of these volumes reflects the entire tumor tissue expressing somatostatin receptor type 2 and was defined as total volume (TV).

\section{Statistical analysis}

Statistical analyses were performed using SPSS 20.0 software (SPSS). Results are expressed as mean \pm standard deviation (s.D.) unless otherwise indicated. For group comparisons, the independent Student's t-test was used to analyze the differences in continuous variables, and the chi-square test was used to analyze differences in categorical variables. The Pearson product was used for analysis of correlations

Table 1 Study cohort clinical characteristics $(n=232)$.

\begin{tabular}{lc}
\hline Primary tumor $n(\%)$ & \\
PNET & $112(48.3 \%)$ \\
SINET & $74(31.9 \%)$ \\
Unknown primary & $16(6.9 \%)$ \\
Other* & $30(12.9 \%)$ \\
Genetic syndromes $n(\%)$ & \\
MEN-1 & $39(16.8 \%)$ \\
VHL & $28(12.1 \%)$ \\
NF-1 & $1(0.4 \%)$ \\
Cowden & $1(0.4 \%)$ \\
Functional status $n(\%)$ & \\
Nonfunctional & $121(52.2 \%)$ \\
Insulinoma & $12(5.2 \%)$ \\
Gastrinoma & $16(6.9 \%)$ \\
Carcinoid syndrome & $79(34.1 \%)$ \\
Other hormone production & $4(1.7 \%)$ \\
WHO tumor grade & \\
G1 & $51(52.0 \%)$ \\
G2 & $42(42.9 \%)$ \\
G3 & $5(5.1 \%)$ \\
Metastases $n$ (\%) & \\
Any & $134(57.8 \%)$ \\
Lymph nodes & $84(36.2 \%)$ \\
Liver & $83(35.8 \%)$ \\
Bones & $32(13.8 \%)$ \\
Lungs & $12(5.2 \%)$ \\
Treatment & \\
SSA $n$ (\%) & $79(34.3 \%)$ \\
History of surgical resection of NETs before & $133(57.3 \%)$ \\
68Ga-DOTATATE PET imaging & $185(79.7 \%)$ \\
Pathological uptake on ${ }^{68}$ Ga-DOTATATE PET & \\
\hline & \\
\hline
\end{tabular}

*Consisted of gastric $(n=6)$, duodenal $(n=10)$, rectal $(n=6)$, appendiceal $(n=1)$, large intestine $(n=2)$, bronchial $(n=3)$, thymic $(n=1)$ and bladder $(n=1)$ NETs. ^Only a subset of patients had WHO tumor grade status available.

MEN-1, multiple endocrine neoplasia syndrome type 1; NF-1, neurofibromatosis type 1; PNET, pancreatic neuroendocrine tumor; SINET, small-intestine neuroendocrine tumor; SSA, somatostatin analogues; VHL, von Hippel-Lindau disease. 
between variables. Receiver-operating characteristic (ROC) curve analysis was performed to assess the accuracy of 24-h urinary 5-HIAA and plasma CgA levels for indicating metastatic SINET. Both TV and biochemical biomarker levels were logarithmically transformed to approximate normality. Non-parametric tests were used as appropriate, including Mann-Whitney $U$ for continuous variables, and Fisher's exact test for categorical variables. The $P$ value for significance was set at less than 0.05 .

\section{Results}

The study cohort consisted of 232 patients with a mean age of $54.3 \pm 14.1$ years, mean body mass index of $28.9 \pm 6.8 \mathrm{~kg} /$ $\mathrm{m}^{2}$ and $130(56.0 \%)$ were females. The sites of NETs were PNET $(n=112)$, SINET $(n=74)$ and tumors of unknown primary $(n=16)$. NEToOL consisted of gastric $(n=6)$, duodenal $(n=10)$, rectal $(n=6)$, appendiceal $(n=1)$, large intestine $(n=2)$, bronchial $(n=3)$, thymic $(n=1)$ and bladder $(n=1)$ NETs. The study cohort clinical characteristics are summarized in Table 1. Mean biomarkers levels, normal reference ranges and percentage of patients with elevated biomarkers levels are summarized in Table 2. Correlation analysis between ${ }^{68} \mathrm{Ga}$-DOTATATE TV and biochemical biomarkers are summarized in Supplementary Table 1(see section on supplementary data at the end of the article).

\section{Tumor burden measurement by ${ }^{68} \mathrm{Ga}$-DOTATATE TV}

The mean ${ }^{68} \mathrm{Ga}$-DOTATATE TV was $69.2 \pm 152.1 \mathrm{~mL}$, and mean SUVmax was 69.1 $\pm 50.9 .{ }^{68} \mathrm{Ga}$-DOTATATE TV positively correlated with 24-h urinary 5-HIAA levels $(r=0.5, P<0.001)$ and plasma CgA levels $(r=0.4$, $P=0.001)$. Patients with metastatic disease had higher 24-h 5-HIAA levels $(14.8 \pm 29.7$ vs $4.8 \pm 2.2 \mathrm{mg} / 24 \mathrm{~h}$, $P<0.001)$, plasma gastrin levels $(252 \pm 863$ vs $74 \pm 120 \mathrm{pg} /$ $\mathrm{mL}, \quad P=0.02)$ and plasma CgA levels $(382 \pm 1030$ vs
$112 \pm 255 \mathrm{ng} / \mathrm{mL}, P=0.002)$ compared to those in patients without metastatic disease respectively.

\section{Tumor burden measurement in patients with PNET}

Patients with PNETs $(n=112)$ had mean ${ }^{68} \mathrm{Ga}$-DOTATATE TV of $71.3 \pm 167.5 \mathrm{~mL}$ and mean SUVmax of $85.5 \pm 58.3$. Sixty-two patients with PNETs (55.4\%) had metastatic disease at evaluation. Among patients with PNETs, tumor burden as measured by ${ }^{68} \mathrm{Ga}$-DOTATATE TV positively correlated with plasma CgA levels $(r=0.6, P=0.001$, Spearman's rho), and plasma NSE levels had a similar trend $(r=0.4, P=0.05)$. Similar trend was found for plasma NSE levels among patients with metastatic PNETs $(r=0.5, P=0.07)$.

Among patients with multiple endocrine neoplasia type-1 (MEN-1) with PNETs, ${ }^{68} \mathrm{Ga}$-DOTATATE TV positively correlated with plasma glucagon $(r=0.5, P=0.01$, Fig. 1 A) and PP levels $(r=0.5, P=0.049)$. In patients with von Hippel-Lindau (VHL), ${ }^{68} \mathrm{Ga}$-DOTATATE TV positively correlated with plasma VIP $(r=0.5, P=0.02$, Fig. 1B) and PP levels ( $r=0.7, P<0.001$, Fig. 1C).

Patients with WHO 2010 G1 PNETs had positive correlation between TV and plasma glucagon levels ( $n=22, r=0.5, P=0.02)$ that was not significant in patients with G2 PNETs $(n=16)$. In 16 patients with gastrinomas of either pancreatic or duodenal location, ${ }^{68} \mathrm{Ga}$-DOTATATE TV had strong positive correlation with plasma gastrin levels $(r=0.8, P=0.001)$.

\section{Tumor burden measurement in patients with SINET}

In patients with SINET $(n=74)$, the mean ${ }^{68} \mathrm{Ga}$-DOTATATE TV and SUVmax were $74.1 \pm 133.5 \mathrm{~mL}$ and $49.1 \pm 25.9$ respectively. 24-h urinary 5-HIAA $(P<0.001)$ levels were higher among patients with distant metastases compared with those in patient without metastases.

Table 2 Neuroendocrine tumor biomarker levels distribution in study cohort.

\begin{tabular}{|c|c|c|c|c|}
\hline Biomarkers & $\begin{array}{l}\text { Upper limit of normal } \\
\text { range }\end{array}$ & $n$ & Mean \pm s.D. & $\begin{array}{l}\text { n (\%) of patients with } \\
\text { elevated biomarkers* }\end{array}$ \\
\hline 24-h urinary 5-HIAA levels & $\leq 8 \mathrm{mg} / 24 \mathrm{~h}$ & 208 & $10.5 \pm 22.9$ & $48(23.1 \%)$ \\
\hline Plasma chromogranin A levels ${ }^{+}$ & $\leq 93 \mathrm{ng} / \mathrm{mL}$ & 83 & $237 \pm 712$ & $24(28.9 \%)$ \\
\hline Plasma NSE levels & $\leq 15 \mathrm{ng} / \mathrm{mL}$ & 81 & $9.4 \pm 1.9$ & $6(7.4 \%)$ \\
\hline Plasma gastrin levels & $\leq 100 \mathrm{pg} / \mathrm{mL}$ & 219 & $176 \pm 661$ & $42(19.2 \%)$ \\
\hline Plasma glucagon levels & $\leq 80 \mathrm{pg} / \mathrm{mL}$ & 220 & $41.4 \pm 42.0$ & $25(11.4 \%)$ \\
\hline Plasma VIP levels & $\leq 75 \mathrm{pg} / \mathrm{mL}$ & 169 & $48.0 \pm 117.0$ & $8(4.7 \%)$ \\
\hline Plasma PP levels & $\leq 291 \mathrm{pg} / \mathrm{mL}$ & 199 & $228 \pm 493$ & $28(14.1 \%)$ \\
\hline
\end{tabular}

www.eje-online.org 

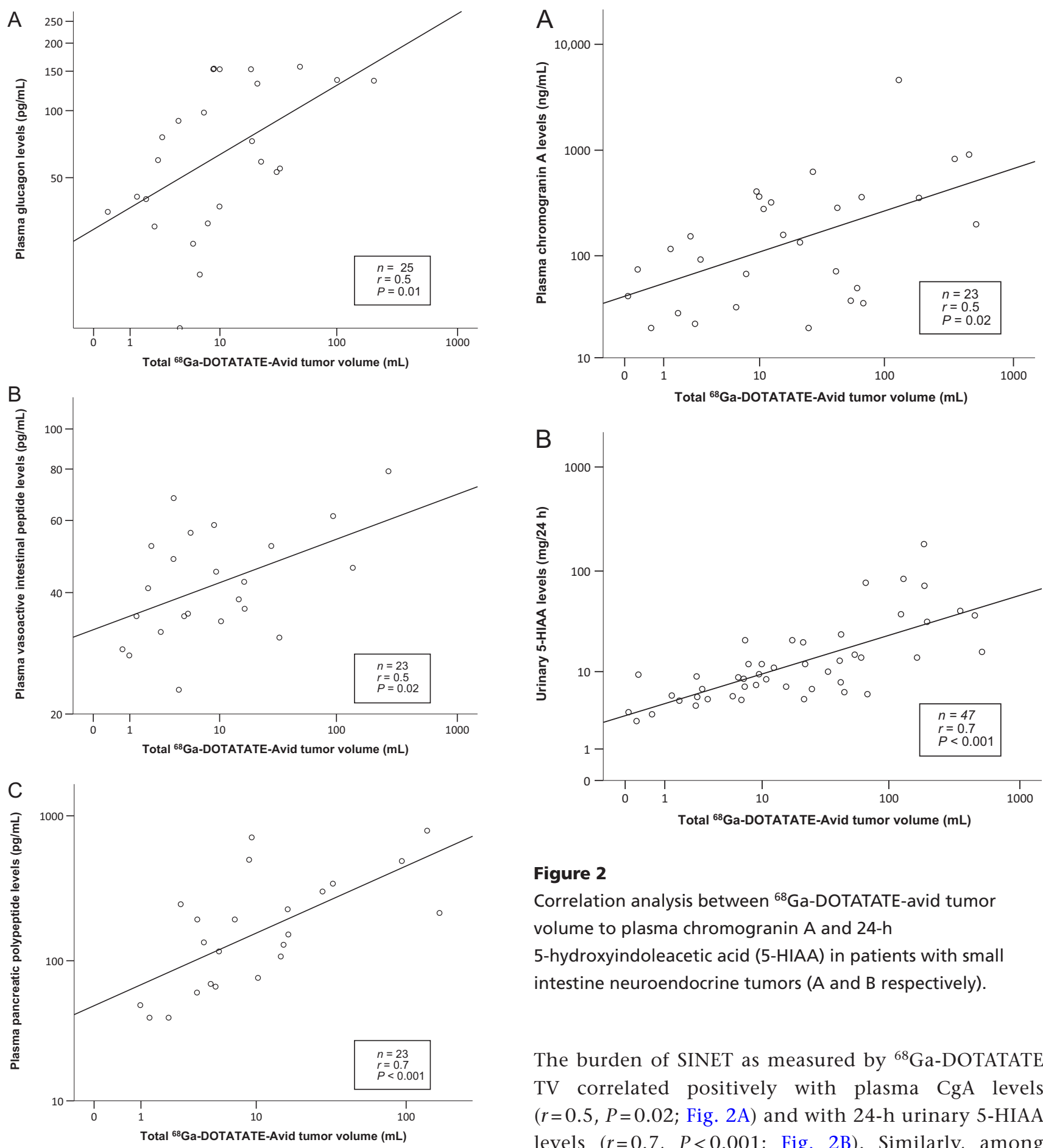

Figure 2

Correlation analysis between ${ }^{68} \mathrm{Ga}$-DOTATATE-avid tumor volume to plasma chromogranin A and 24-h

5-hydroxyindoleacetic acid (5-HIAA) in patients with small intestine neuroendocrine tumors ( $A$ and $B$ respectively).

The burden of SINET as measured by ${ }^{68} \mathrm{Ga}$-DOTATATE TV correlated positively with plasma CgA levels $(r=0.5, P=0.02$; Fig. $2 \mathrm{~A})$ and with 24 -h urinary 5-HIAA levels ( $r=0.7, P<0.001$; Fig. 2B). Similarly, among the subgroup of patients with metastatic SINET,

Figure 1

Correlation analysis between ${ }^{68} \mathrm{Ga}$-DOTATATE-avid tumor volume of pancreatic neuroendocrine tumors to plasma glucagon levels among patients with multiple endocrine neoplasia syndrome type-1 (A), plasma vasoactive intestinal peptide and pancreatic polypeptide levels among patients with von Hippel-Lindau disease ( $B$ and $C$ respectively). ${ }^{68} \mathrm{Ga}$-DOTATATE TV had positive correlation with CgA levels $(r=0.5, P=0.02)$ and with 24 -h urinary 5-HIAA levels $(r=0.7, P<0.001)$. In patients with carcinoid syndrome $(n=32),{ }^{68} \mathrm{Ga}$-DOTATATE TV correlated with 24-h urinary 5-HIAA $(r=0.7, P<0.001)$ and plasma CgA levels $(r=0.5, P=0.02)$, whereas in patients without carcinoid syndrome, ${ }^{68} \mathrm{Ga}$-DOTATATE TV 
A
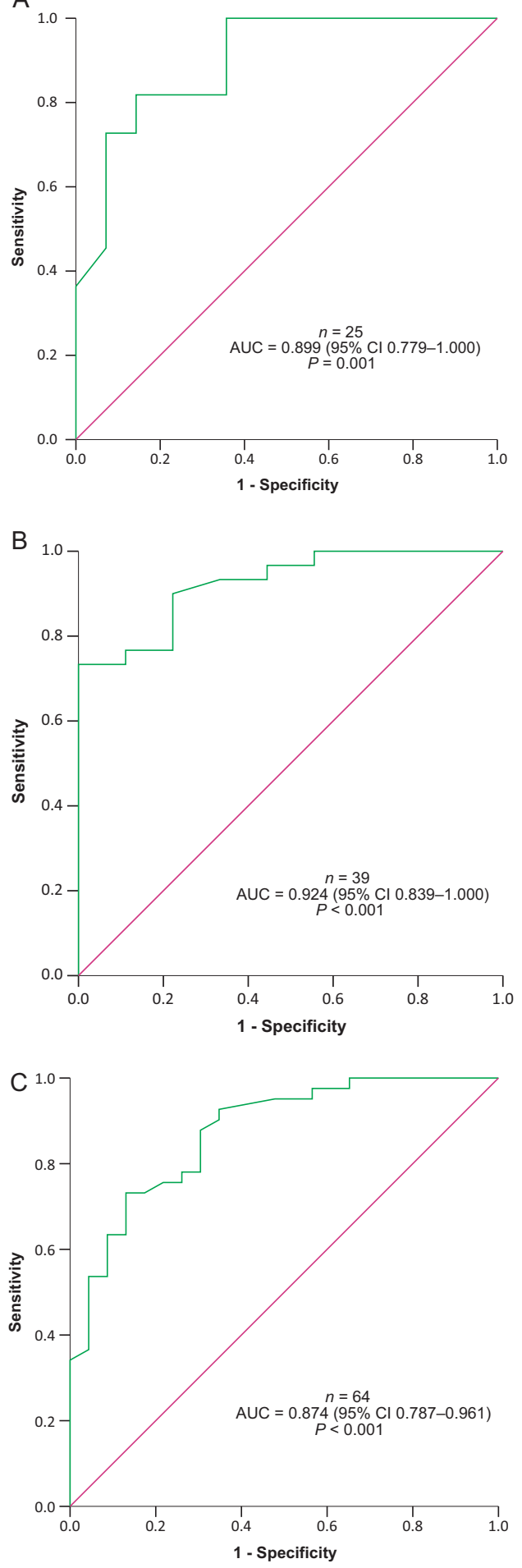

Figure 3

Receiver-operating characteristic (ROC) analysis for 24-h urinary 5-hydroxyindoleacetic acid (5-HIAA) levels for predicting metastatic disease in subjects with small intestine neuroendocrine tumors (SINETs). Patients with (A) and without (B) somatostatin analogues treatment and including all SINET patients (C). correlated only with 24 -h urinary 5 -HIAA levels $(n=15$, $r=0.9, P<0.001)$.

Urinary 5-HIAA levels $\geq 8.1 \mathrm{mg} / 24 \mathrm{~h}$ (upper limit of normal range, $8 \mathrm{mg} / 24 \mathrm{~h}$ ) predicted metastatic disease based on ${ }^{68} \mathrm{Ga}$-DOTATATE PET/CT among subjects with SINET that were not treated with somatostatin analogues, with a sensitivity of $81.8 \%$ and a specificity of $85.7 \%$, and positive and negative predictive values of $81.8 \%$ and $85.7 \%$ respectively $(P=0.001$, Fig. $3 \mathrm{~A})$. This was true also among patients treated with somatostatin analogues (Fig. 3B) and among all subjects with SINET (Fig. 3C). In contrast, ROC curve analysis revealed limited utility of plasma CgA levels for predicting metastatic SINET (area under the curve $=0.668,95 \%$ confidence interval 0.548 $0.788, P=0.008)$.

\section{Tumor burden measurement in NEToUP and NEToOL}

Among sixteen patients with NEToUP, correlation analysis revealed strong correlation between ${ }^{68} \mathrm{Ga}$-DOTATATE TV and 24-h urinary 5-HIAA levels $(r=0.8, P=0.002)$. Subjects with NEToOL $(n=30)$ had positive correlation between ${ }^{68} \mathrm{Ga}$-DOTATATE TV and 24-h 5-HIAA $(r=0.7, P=0.02$, Spearman's rho).

The analyses were repeated after excluding patients treated with somatostatin analogues with findings similar to those described for the whole study cohort analysis. Among subjects with NEToOL, excluding patients with somatostatin analogue treatment revealed a correlation between ${ }^{68} \mathrm{Ga}$-DOTATATE TV and plasma PP levels $(n=7$, $r=0.8, P=0.03)$.

\section{Discussion}

In this study, we analyzed the utility of seven biomarkers for assessing NET burden using a novel tumor burden measurement approach based on ${ }^{68} \mathrm{Ga}$-DOTATATE TV in a large cohort of patients with NET. ${ }^{68} \mathrm{Ga}$-DOTATATE TV correlated strongly with urinary 5-HIAA and with plasma CgA among patients with SINETs. In addition, MEN-1 patients with PNETs had correlation between tumor burden and plasma glucagon levels, and subjects with VHL disease had correlation between ${ }^{68} \mathrm{Ga}$-DOTATATE TV and plasma PP and VIP levels. Finally, urinary 5-HIAA levels $\geq 8.1 \mathrm{mg} / 24 \mathrm{~h}$ were associated with metastatic disease in patients with SINET, independent of somatostatin analogue treatment.

Tumor biomarkers measurement are the cornerstone of NET diagnosis (20) and are essential for surveillance after various treatment modalities are carried out. Plasma 
CgA levels reflect tumor burden in non-functioning PNETs (21), and high urinary 5-HIAA was found mainly in patients with metastatic SINET (22) and is associated with carcinoid heart disease (23). Indeed, the current guidelines for NET management recommend the use of urinary 5-HIAA for SINET diagnosis, and the measurement of CgA and NSE for the diagnosis of PNET $(4,5)$. The current study provides data to support these recommendations of using specific NET biomarkers based on the primary tumor site and that elevated values of urinary 5-HIAA for SINET often indicate the presence of metastatic disease, which may not be seen by conventional anatomic imaging studies.

Somatostatin receptor-based PET/CT is a very sensitive imaging modality to detect NETs $(10,24,25) .{ }^{68} \mathrm{Ga}$-labeled somatostatin analogue PET/CT is more sensitive for NET localization $(5,10)$, is faster and requires lower radiation dose (26) compared with Octreoscan. Hence, in the current study, disease extent was assessed by the quantification of whole-body ${ }^{68} \mathrm{Ga}$-DOTATATE pathological uptake. Thus, we believe our analysis of the levels of a comprehensive panel of NET biomarkers and NET disease burden more accurately reflects the diagnostic and surveillance clinical utility of biomarkers in patients with NET.

Total ${ }^{68} \mathrm{Ga}$-DOTATATE uptake in NETs was reported in the past in a series of patients, but not in specific subtypes of NET, and no correlation analysis with tumor biomarkers was reported $(12,13)$. Moreover, the correlations between tumor biomarkers and tumor burden reported in the past have been based on anatomical imaging (21), counting the number of lesions (10), the presence/absence of liver metastases (27) and not based on accurate total tumor volume measurements, as performed in the current analysis. The strong correlation between 24-h urinary 5-HIAA levels and ${ }^{68} \mathrm{Ga}$-DOTATATE TV in SINET is not surprising (22) and might explain the strong correlation between total volume measurements and 5-HIAA among patients with NEToUP, as these tumors arise mainly from the small intestine but are not detected (28).

Our findings have important clinical implications. First, baseline 5 -HIAA levels $\geq 8.1 \mathrm{mg} / 24 \mathrm{~h}$ should prompt evaluation for metastatic disease in patients with SINET. Second, among patients with PNETs associated with hereditary syndromes, PP may be used as a biomarker in patients with MEN-1, while glucagon and VIP can be used as biomarkers in patients with VHL.

Our analysis has some limitations. First, the vast majority of the patients in the current study had gastroenteropancreatic (GEP) NETs, limiting the ability to make strong conclusions about biomarkers utility among patients with primary tumors at other sites. Second, although the analysis was performed by an experienced nuclear medicine specialist who was blinded to the clinical context, interobserver variation was not tested for in the current study and should be evaluated in future studies. Third, plasma CgA levels may be elevated in patients with atrophic gastritis and can lead to positive results, and every patient in the current study did not have endoscopy and biopsy to determine the presence of this. Finally, the biochemical biomarkers in the current analysis were performed using a single method per biomarker. As there might be a difference in the measured levels between different analytical methods, the study results may only apply to the methods used in our study and other analytical methods would need to be studied in the future.

In conclusion, our data support the use of specific NET markers based on the site of the primary NET and the presence of hereditary syndrome-associated NET. High levels of 24-h urinary 5-HIAA commonly indicate the presence of metastatic disease in patients with SINET. The heterogeneity of patients with NET necessitates careful matching of biochemical biomarker for each patient according to both tumor and patient characteristics and might justify personalized biomarker panel evaluation for tumor burden assessment.

\section{Supplementary data}

This is linked to the online version of the paper at http://dx.doi.org/10.1530/ EJE-16-1079.

Declaration of interest

The authors declare that there is no conflict of interest that could be perceived as prejudicing the impartiality of this study.

\section{Funding}

This study was supported by the Intramural Research Programs of the Center for Cancer Research, National Cancer Institute, National Institutes of Health.

\section{References}

1 Pavel M, O’Toole D, Costa F, Capdevila J, Gross D, Kianmanesh R, Krenning E, Knigge U, Salazar R, Pape U-F et al. ENETS consensus guidelines update for the management of distant metastatic disease of intestinal, pancreatic, bronchial neuroendocrine neoplasms (NEN) and NEN of unknown primary site. Neuroendocrinology 2016103 172-185. (doi:10.1159/000443167)

2 Lawrence B, Gustafsson BI, Chan A, Svejda B, Kidd M \& Modlin IM. The epidemiology of gastroenteropancreatic neuroendocrine tumors. Endocrinology and Metabolism Clinics of North America 201140 1-18. (doi:10.1016/j.ecl.2010.12.005)

3 Frilling A, Modlin IM, Kidd M, Russell C, Breitenstein S, Salem R, Kwekkeboom D, Lau W, Klersy C, Vilgrain V et al. Recommendations for management of patients with neuroendocrine liver metastases. Lancet Oncology 201415 e8-e21. (doi:10.1016/S1470-2045(13)70362-0) 
4 Falconi M, Eriksson B, Kaltsas G, Bartsch DK, Capdevila J, Caplin M, Kos-Kudla B, Kwekkeboom D, Rindi G, Klöppel G et al. ENETS consensus guidelines update for the management of patients with functional pancreatic neuroendocrine tumors and non-functional pancreatic neuroendocrine tumors. Neuroendocrinology 2016103 153-171. (doi:10.1159/000443171)

5 Niederle B, Pape U-F, Costa F, Gross D, Kelestimur F, Knigge U, Öberg K, Pavel M, Perren A, Toumpanakis C et al. ENETS consensus guidelines update for neuroendocrine neoplasms of the jejunum and ileum. Neuroendocrinology 2016103 125-138. (doi:10.1159/000443170)

6 Arnold R, Chen Y-J, Costa F, Falconi M, Gross D, Grossman AB, Hyrdel R, Kos-Kudła B, Salazar R, Plöckinger U et al. ENETS consensus guidelines for the standards of care in neuroendocrine tumors: follow-up and documentation. Neuroendocrinology 200990 227-233. (doi:10.1159/000225952)

7 Oberg K, Modlin IM, De Herder W, Pavel M, Klimstra D, Frilling A, Metz DC, Heaney A, Kwekkeboom D, Strosberg J et al. Consensus on biomarkers for neuroendocrine tumour disease. Lancet Oncology 2015 16 e435-e446. (doi:10.1016/S1470-2045(15)00186-2)

8 Santhanam P, Chandramahanti S, Kroiss A, Yu R, Ruszniewski P, Kumar R \& Taïeb D. Nuclear imaging of neuroendocrine tumors with unknown primary: why, when and how? European Journal of Nuclear Medicine and Molecular Imaging 201542 1144-1155. (doi:10.1007/ s00259-015-3027-4)

9 Schreiter NF, Bartels A-M, Froeling V, Steffen I, Pape U-F, Beck A, Hamm B, Brenner W \& Röttgen R. Searching for primaries in patients with neuroendocrine tumors (NET) of unknown primary and clinically suspected NET: evaluation of Ga-68 DOTATOC PET/CT and In-111 DTPA octreotide SPECT/CT. Radiology and Oncology 201448 339-347.

10 Sadowski SM, Neychev V, Millo C, Shih J, Nilubol N, Herscovitch P, Pacak K, Marx SJ \& Kebebew E. Prospective study of 68Ga-DOTATATE positron emission tomography/computed tomography for detecting gastro-entero-pancreatic neuroendocrine tumors and unknown primary sites. Journal of Clinical Oncology 201634 588-596. (doi:10.1200/JCO.2015.64.0987)

11 Deroose CM, Hindie E, Kebebew E, Goichot B, Pacak K, Taieb D \& Imperiale A. Molecular imaging of gastroenteropancreatic neuroendocrine tumors: current status and future directions. Journal of Nuclear Medicine 201657 1949-1956.

12 Abdulrezzak U, Kurt YK, Kula M \& Tutus A. Combined imaging with 68Ga-DOTA-TATE and 18F-FDG PET/CT on the basis of volumetric parameters in neuroendocrine tumors. Nuclear Medicine Communications 201637 874-881. (doi:10.1097/ MNM.0000000000000522)

13 Cieciera M, Kratochwil C, Moltz J, Kauczor HU, Holland Letz T, Choyke P, Mier W, Haberkorn U \& Giesel FL. Semi-automatic 3D-volumetry of liver metastases from neuroendocrine tumors to improve combination therapy with 177Lu-DOTATOC and 90Y-DOTATOC. Diagnostic and Interventional Radiology 201622 201-206. (doi:10.5152/dir.2015.15304)

14 Sato Y, Hashimoto S, Mizuno K-I, Takeuchi M \& Terai S. Management of gastric and duodenal neuroendocrine tumors. World Journal of Gastroenterology 201622 6817-6828. (doi:10.3748/wjg.v22.i30.6817)

15 Klimstra DS, Modlin IR, Coppola D, Lloyd RV \& Suster S. The pathologic classification of neuroendocrine tumors: a review of nomenclature, grading, and staging systems. Pancreas 201039 707-712. (doi:10.1097/MPA.0b013e3181ec124e)

16 Roberts RO, Aakre JA, Cha RH, Kremers WK, Mielke MM, Velgos SN, Geda YE, Knopman DS \& Petersent RC. Association of Pancreatic
Polypeptide with Mild Cognitive Impairment Varies by APOE 4I Allele. Frontiers in Aging Neuroscience 2015. (doi:10.3389/ fnagi.2015.00172)

17 Kroll CA, Magera MJ, Helgeson JK, Matern D \& Rinaldo P. Liquid Chromatographic-Tandem Mass Spectrometric Method for the Determination of 5-Hydroxyindole-3-acetic Acid in Urine. Clinical Chemistry 200248 2049-2051.

18 Foster B, Bagci U, Mansoor A, Xu Z \& Mollura DJ. A review on segmentation of positron emission tomography images. Computers in Biology and Medicine 201450 76-96. (doi:10.1016/j. compbiomed.2014.04.014)

19 Etchebehere EC, Araujo JC, Fox PS, Swanston NM, Macapinlac HA \& Rohren EM. Prognostic factors in patients treated with 223Ra: the role of skeletal tumor burden on baseline 18F-fluoride PET/CT in predicting overall survival. Journal of Nuclear Medicine $2015 \mathbf{5 6}$ 1177-1184. (doi:10.2967/jnumed.115.158626)

20 Ardill JES \& Erikkson B. The importance of the measurement of circulating markers in patients with neuroendocrine tumours of the pancreas and gut. Endocrine-Related Cancer 200310 459-462. (doi:10.1677/erc.0.0100459)

21 Han X, Zhang C, Tang M, Xu X, Liu L, Ji Y, Pan B \& Lou W. The value of serum chromogranin $A$ as a predictor of tumor burden, therapeutic response, and nomogram-based survival in well-moderate nonfunctional pancreatic neuroendocrine tumors with liver metastases. European Journal of Gastroenterology and Hepatology 2015 27 527-535. (doi:10.1097/MEG.0000000000000332)

22 Norheim I, Oberg K, Theodorsson-Norheim E, Lindgren PG, Lundqvist G, Magnusson A, Wide L \& Wilander E. Malignant carcinoid tumors. An analysis of 103 patients with regard to tumor localization, hormone production, and survival. Annals of Surgery 1987206 115-125. (doi:10.1097/00000658-198708000-00001)

23 Zuetenhorst JM, Bonfrer JMGM, Korse CM, Bakker R, van Tinteren H \& Taal BG. Carcinoid heart disease: the role of urinary 5-hydroxyindoleacetic acid excretion and plasma levels of atrial natriuretic peptide, transforming growth factor-beta and fibroblast growth factor. Cancer 200397 1609-1615.

24 Deppen SA, Blume J, Bobbey AJ, Shah C, Graham MM, Lee P, Delbeke D \& Walker RC. 68Ga-DOTATATE compared with 111In-DTPA-octreotide and conventional imaging for pulmonary and gastroenteropancreatic neuroendocrine tumors: a systematic review and meta-analysis. Journal of Nuclear Medicine 201657 872-878. (doi:10.2967/jnumed.115.165803)

25 Skoura E, Michopoulou S, Mohmaduvesh M, Panagiotidis E, Al Harbi M, Toumpanakis C, Almukhailed O, Kayani I, Syed R, Navalkissoor S et al. The impact of 68Ga-DOTATATE PET/CT imaging on management of patients with neuroendocrine tumors: experience from a national referral center in the United Kingdom. Journal of Nuclear Medicine 201657 34-40. (doi:10.2967/jnumed.115.166017)

26 Baumann T, Rottenburger C, Nicolas G \& Wild D. Gastroenteropancreatic neuroendocrine tumours (GEP-NET) imaging and staging. Best Practice and Research Clinical Endocrinology and Metabolism 201630 45-57. (doi:10.1016/j.beem.2016.01.003)

27 Nölting S, Kuttner A, Lauseker M, Vogeser M, Haug A, Herrmann KA, Hoffmann JN, Spitzweg C, Göke B \& Auernhammer CJ. Chromogranin a as serum marker for gastroenteropancreatic neuroendocrine tumors: a single center experience and literature review. Cancers 20124 141-155. (doi:10.3390/cancers4010141)

28 Wang SC, Parekh JR, Zuraek MB, Venook AP, Bergsland EK, Warren RS $\&$ Nakakura EK. Identification of unknown primary tumors in patients with neuroendocrine liver metastases. Archives of Surgery 2010 145 276-280. (doi:10.1001/archsurg.2010.10)
Received 30 December 2016

Revised version received 8 February 2017

Accepted 14 February 2017 